\title{
Dynamic Diffractive Patterns in Helix-Inverting Cholesteric Liquid Crystals
}

\author{
Alexander Ryabchun, ${ }^{* \dagger, \ddagger(\odot) ~ D m i t r y ~ Y a k o v l e v, ~}{ }^{\S}$ Alexey Bobrovsky, ${ }^{\|}$and Nathalie Katsonis ${ }^{\dagger}$ \\ ${ }^{\dagger}$ Bio-inspired and Smart Materials, MESA+ Institute for Nanotechnology, University of Twente, P.O. Box 207, 7500 AE Enschede, \\ The Netherlands \\ ${ }^{\ddagger}$ Fraunhofer Institute for Applied Polymer Research, Geiselbergstr. 69, 14476 Potsdam, Germany \\ ${ }^{\S}$ Physics Department, Saratov State University, Astrakhanskaya 83, Saratov 410012, Russia \\ "Chemistry Department, Moscow State University, Lenin Hills 1, Moscow 119991, Russia
}

\section{Supporting Information}

ABSTRACT: The future of adaptive materials will rely on transduction of molecular motion across increasing length scales, up to the macroscopic and functional level. In this context, liquid crystals have emerged as a promising amplification medium, in view of their long-range order and high sensitivity to external stimuli, and in particular, chiral liquid crystals have demonstrated widely tunable optical

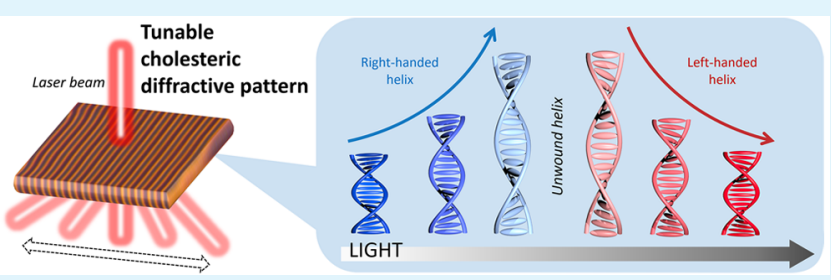
properties and invertible handedness. Here, we demonstrate that by applying weak electric fields, regular, periodic and light-tunable patterns can be formed spontaneously in cholesteric liquid crystals. These patterns can be used as light-tunable diffraction gratings for which the period, the diffraction efficiency, and the in-plane orientation of grating vector can be controlled precisely, reversibly, and independently. Such a photoregulation allows generating a variety of one- and two-dimensional complex diffractive patterns in a single material. Our data are also supported by modeling and theoretical calculations. Overall, the fine tunability of cholesteric materials doped with artificial molecular switches makes them attractive for optics and photonics.

KEYWORDS: chiral liquid crystals, diffraction gratings, molecular switches, helix inversion, light

\section{INTRODUCTION}

Incorporating molecular motors and switches in dynamic functional systems requires strategies to transfer their molecular-scale operation up to the macroscopic level. ${ }^{1,2}$ Arguably, the most effective approaches have involved incorporating artificial molecular motors and switches in soft matter systems $s^{3,4}$ and in particular in liquid crystals (LCs) ${ }^{5}$ and LC polymer networks, ${ }^{6}$ in view of their long range order and anisotropic character. Examples of macroscopic effects include the rotation of microscopic objects under the action of chiral molecular motors ${ }^{7}$ and the shape transformation of LC polymer photoactuators. $^{8,9}$

Among all LCs, cholesteric LCs (CLCs) are particularly relevant media to amplify the operation of artificial molecular motors and switches. ${ }^{10}$ Their periodic helical organization and high stimuli responsiveness make them promising field-driven materials for applications ranging from soft robotics to optics, photonics, lasers, sensors, and so forth. ${ }^{11,12}$ A salient feature of cholesteric materials is their aptitude to reflect light selectively because the nanoscale periodicity of their helical structure supports a Bragg-like reflection. This property has been investigated in-depth in the last years in view of its potential applications ${ }^{11}$ and will not be discussed hereafter. In contrast, here we discuss another optical material that can be prepared by applying an electric field on thin film of CLCs, namely, a light-responsive material that features a periodic in-plane modulation of the LC director. Such an in-plane modulation can mediate the use of CLCs as tunable diffractive optical elements, ${ }^{13-20}$ and recently it has been suggested that it could be used as anticounterfeiting two-dimensional (2D) barcodes. $^{21}$ To date, linear, ${ }^{22,23}$ circular, $^{24}$ zigzag, $^{25}$ square, ${ }^{2,26}$ and hexagonal $^{27}$ modulations could be engineered depending on the geometrical characteristics of the cholesteric helix, its confinement, and the boundary conditions. These structures can be stabilized in the glassy state of LC side-chain polymers ${ }^{28}$ or by the formation of cross-polymerized polymer networks. $^{29,30}$ In addition to the practical significance of such in-plane patterns for optics, we also envision their relevance to other applied fields. For example, it has been shown recently that these electro-induced patterns can be used to manipulate colloidal particles. ${ }^{31}$ Electro-induced structuring can also be useful for the creation of novel shape-shifting polymers materials or photo-actuators with enhanced actuation modes $^{32}$ as well as photocontrollable dynamic surfaces. ${ }^{33}$ However, controlling the parameters of these patterns in situ and in a wide range remains a challenge. We anticipate that

Received: December 26, 2018

Accepted: February 19, 2019

Published: February 19, 2019 
a)

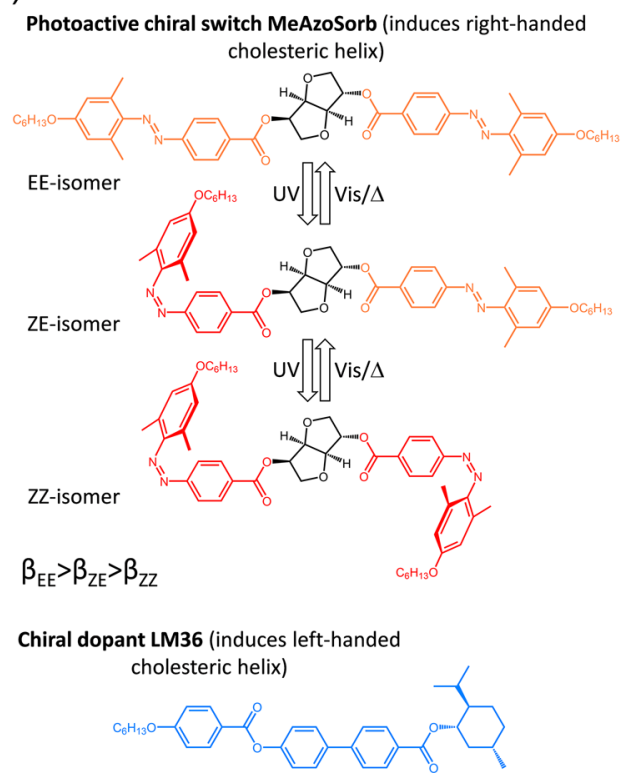

b)
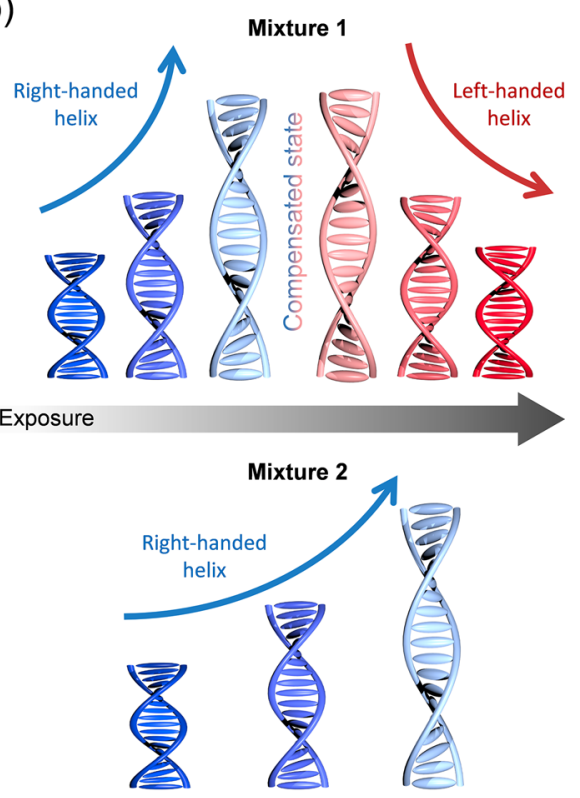

Figure 1. (a) Chemical structures of the used chiral dopants and photo-isomerization scheme of photoactive chiral switch MeAzoSorb. (b) Schematic representation of two CLCs that are used here. CLC mixture 1 (top panel) exhibits inversion of cholesteric handedness and CLC mixture 2 (bottom panel) shows only unwinding of the helix upon irradiation with light.

improving control overdynamic chiral structures can give new impetus to the development of these materials.

Reports on electro-induced patterns in CLCs have remained fragmented and their classification was not unified, likely because conventional CLCs do not allow tuning the confinement ratio in a wide range. In our work, the helical pitch can be tuned over a large range of values, and thus the confinement of the cholesteric helix can undergo large in situ variations too, also with the possibility of reversible variation of supramolecular chirality, which is why a large number of onedimensional (1D) and 2D electro-induced patterns can be obtained on the basis of only one material. Our work provides a comprehensive experimental investigation on the molecular rules that drive the formation of these structures. Moreover, the structural and optical properties of the diffractive patterns have been predicted with good accuracy by numerical simulations which simplify the search for optimal materials, design parameters, and operating modes of devices, as well as the assessment of achievable output characteristics. Overall, the light-tunable in-plane diffractive patterns we describe show perspective in terms of their applications in optics, photonics, and spectroscopy for producing easy-tunable light dispersive elements and beam steering devices.

\section{RESULTS AND DISCUSSION}

2.1. CLCs with a Light-Responsive Helical Pitch. The natural pitch $P_{0}$ of the cholesteric helix is key to determine the geometry of the electro-induced in-plane patterns, and its value depends primarily on the chemical structure of the molecules composing the LC. Typically, CLCs are formed by doping a nematic LC with a chiral dopant. Each dopant/host system is characterized by the helical twisting power, $\beta$, a phenomenological parameter that describes the ability of the dopant to twist the nematic organization

$$
\beta=P_{0}^{-1} / C \cdot e e
$$

where $C$ is the concentration of the chiral dopant and ee is the enantiomeric excess. By convention, $P_{0}$ and $\beta$ are defined as positive values if the native cholesteric helix is right-handed and defined as negative values if the cholesteric helix is lefthanded. The value of $\beta$ is the sum of all individual contributions of the chiral molecules present in the system. The relation between the structure of the chiral dopant structure and the helical twisting power provides a handle to control the period and handedness of the cholesteric helix. ${ }^{34-36}$ For example, in azobenzene-based compounds, irradiation with UV light induces formation a bent-shaped Zisomer having a substantially lower $\beta$ than an initial rod-like Eisomer, and this structural change modifies the period of the cholesteric helix considerably (Figure 1a).

In this work, we use a chiral azobenzene, MeAzoSorb, as a mediator for the pitch modification (Figure 1a). In its stable state, MeAzoSorb induces a right-handed cholesteric helix. Irradiation of this molecule with UV light $(\lambda=365 \mathrm{~nm})$ triggers the formation of a bent-shaped $\mathrm{Z}$-isomer with a lower helical twisting power compared to the initial rod-like Eisomer. Key features of this dopant are its high sensitivity to light, the photo-optical reversibility of the $\mathrm{E}-\mathrm{Z}$ isomerization process, and thermal stability of the Z-isomer as mediated by the lateral methyl substituents. The Z-to-E back isomerization of the switch moieties composing this dopant requires almost $100 \mathrm{~h}$ at $29^{\circ} \mathrm{C}$, in the dark. ${ }^{37}$ The helical twisting power of MeAzoSorb in the stable EE-state is $\sim 61.5 \mu \mathrm{m}^{-1}$.

MeAzoSorb has been used as a dopant to prepare two CLC mixtures with different behaviors under irradiation with light; mixture 1 features a photo-inversion of the cholesteric helix, whereas mixture 2 only features a light-induced pitch modification, as illustrated in Figure $1 \mathrm{~b}$. Table 1 describes the pitch of both CLCs. ${ }^{39}$ Notably, we have used a co-doping approach to preprogram the helix inversion in mixture 1 . In this co-doping approach, a shape-persistent chiral dopant that induces a left-handed helix weakly (LM36, $\beta \approx-10.3 \mu \mathrm{m}^{-1}$ ) is combined with the light-responsive chiral dopant MeAzoSorb 
Table 1. Natural Cholesteric Helix Pitches (in $\mu \mathrm{m}$ ) of CLC Mixtures in Native State $\left(P_{0}\right)$ and in Photostationary States upon UV and Visible Light Exposure $\left(P_{\text {UV-PSs }}\right.$ and $P_{\text {VIS-PSs, }}$ Respectively)

$\begin{array}{cccc} & P_{0} & P_{\text {UV-PSS }} & P_{\text {VIS-PSS }} \\ \text { mixture 1 } & 2.2 & -2.9 & 5 \\ \text { mixture 2 } & 2.5 & 30 & 3.5\end{array}$

that induces a right-helix strongly in the stable state (MeAzoSorb, $\beta \approx+61.5 \mu \mathrm{m}^{-1}$ ). Initially, before irradiation the twisting provided by the EE-isomer of MeAzoSorb overcompensates that of the shape-persistent dopant and the helix is right-handed. Upon irradiation with light, the twisting power of MeAzoSorb decreases by $\mathrm{E}-\mathrm{Z}$ isomerization (Figure 1a), and eventually the effects of the two dopants compensate each other to yield a nontwisted, compensated nematic state. Further irradiation with light leads to helix inversion and, at the photostationary state the cholesteric helix of mixture 1 is lefthanded.

2.2. Electro-Induced Formation of Patterns in Thin Films of CLCs. Before description of the experimental results, in the current section we aim to create a clear picture of the whole variety of electro-induced periodic patterns in CLC layers. Modulated cholesteric patterns able to act as diffractive gratings are usually obtained in cells with planar parallel boundary conditions filled with a CLC material having a positive dielectric anisotropy $\left(\Delta \varepsilon=\varepsilon_{\|}-\varepsilon_{\perp}>0\right)$ at the frequency of driving voltage $(U)$. The LC layer has a planar twisted texture with a twist angle

$$
\Phi=M \pi
$$

where $M$ is an integer with $|M| \geq 1$, the number of half-turns of the cholesteric helix across the layer. We define $\Phi$ and $M$ to be positive if the helical structure is right-handed. According to the Oseen-Frank elastic theory of LCs, to produce the twist configuration with a given $M$ in a cell of a thickness $d$, one should choose the natural pitch $P_{0}$ to satisfy the condition

$$
\operatorname{int}\left(\frac{2 d}{P_{0}}+\frac{1}{2}\right)=M
$$

where $\operatorname{int}(v)$ is the integer part of a number $v$ (the maximum integer not exceeding $v$ ). In terms of the actual helix pitch of the LC structure $P(P \equiv 2 \pi d / \Phi)$, this condition can be reformulated as

$$
\frac{1}{P}-\frac{1}{4}<\frac{d}{P_{0}}<\frac{1}{P}+\frac{1}{4}
$$

At $P_{0}=2 \pi d / \Phi$, the effective pitch $P$ of the thin film in confinement will be equal to the natural pitch $P_{0}$ (the natural, undistorted cholesteric helix). In confinement, the actual helix can be compressed $\left(|P|<\left|P_{0}\right|\right)$ or stretched $\left(|P|>\left|P_{0}\right|\right)$. It is important to note that the properties of modulated structures in CLC layers depend both on $M$ and on the state of the cholesteric helix at zero voltage and on whether it is undistorted, compressed, or stretched. ${ }^{40-42}$

For CLC layers with $|M| \geq 1$ at $\Delta \varepsilon>0$, there is a range of voltage values $U_{\mathrm{TH}-\mathrm{M}}<U<U_{\mathrm{UT}}$ at which the LC pattern is modulated in-plane. Below $U_{\mathrm{TH}-\mathrm{M}}$ and above $U_{\mathrm{UT}}$ the texture remains particularly nonchanged or becomes quasi-homeotropic, respectively. Two field-induced structural transitions leading to in-plane modulated periodic patterns can be distinguished in such cells. One is roughly described as a periodic bending of cholesteric planes called Helfrich-Hurault $(\mathrm{HH})$ deformation $^{43,44}$ (Figure 2a) while the other is a $90^{\circ}$
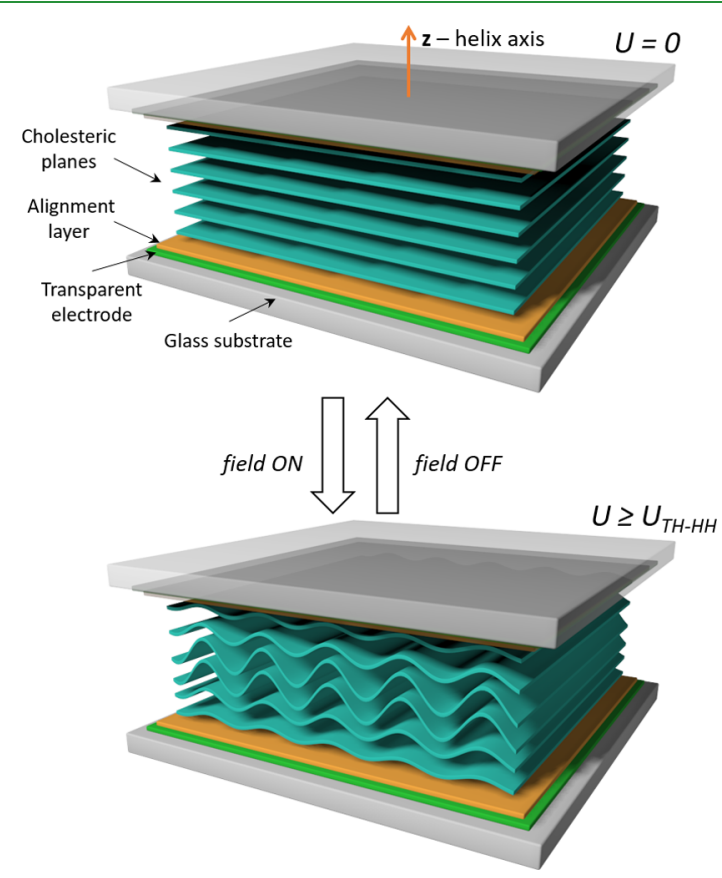

Figure 2. Schematic representation of the $\mathrm{HH}$ deformation (1Dpattern) in a cholesteric layer under the action of electric filed.

rotation of the helical axis. ${ }^{40,45}$ The $\mathrm{HH}$ deformation can be observed in layers with any $M \neq 0$. At $d / P_{0}<1.75, \mathrm{HH}$ deformation gives striped domain structures (1D periodic patterns) ${ }^{42,46}$ with stripes either parallel (Figure 3a on the right) or perpendicular (Figure $3 \mathrm{a}$, at the center) to the rubbing direction. In the case of $\mathrm{HH}$ deformation, the transformation of the ground planar structure begins simultaneously and develops uniformly throughout the switched area of the CLC layer (see Figure S2), due to which this kind of deformation is often referred to as a "developable modulation" (DM).

For layers with $|M| \geq 2$, in a voltage range which commonly covers the range of $\mathrm{HH}$ deformation, the most energetically favorable LC configuration (the configuration corresponding to the global energetic minimum) is a surface-frustrated lyinghelix (SFLH) configuration (Figure 3a, on the left). Line defects that evolve into an SFLH pattern usually nucleate near the edges and spacers of the LC cell, and fold forming stripes. The stripes elongate always parallel to the rubbing direction occupying the whole sample area (Figure S2). This mechanism by which in-plane modulated patterns are formed is also referred to as "growing modulation" (GM). ${ }^{47}$

In layers with $d / P_{0}>2.5$, the $\mathrm{HH}$ domain pattern has the form of square grid (2D-patterns). ${ }^{23,26,29,40,46,48-51}$ In layers with $1.75<d / P_{0}<2.5$, both $1 \mathrm{D}$ - and $2 \mathrm{D}$-periodic patterns are observed and the latter may replace the former by increasing the applied voltage, as will be demonstrated further.

Phototuning $P_{0}$ allows changing both $M$ and the state of the helix. In tested cells with mixture $1(2)$, we can easily tune $M$ from -3 to 2 ( 0 to 2 ) for cells with $d=5 \mu \mathrm{m}$ and from -5 to 4 (0 to 4) for cells with $d=9 \mu \mathrm{m}$ upon light exposure. We anticipate obtaining all kinds of electro-induced patterns 
a)

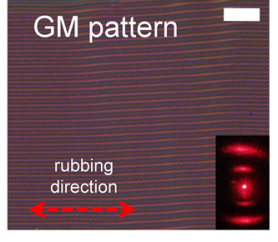

b)
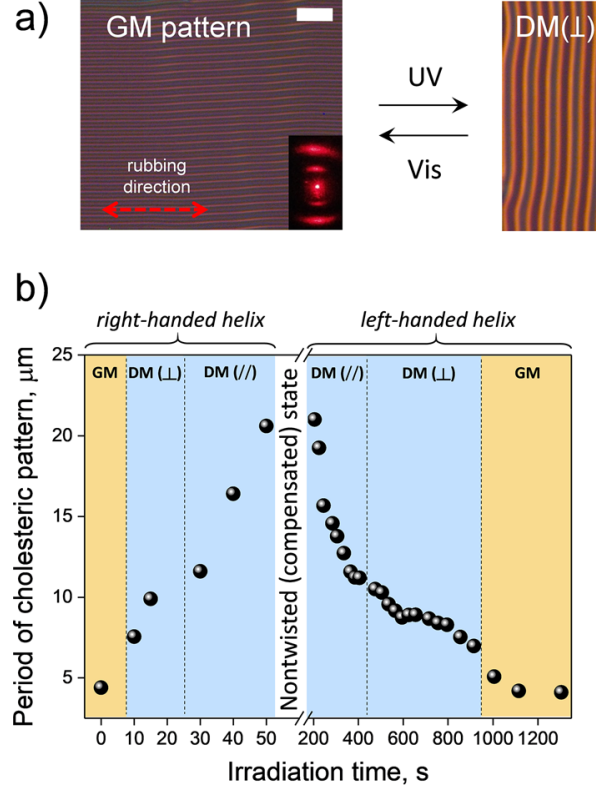
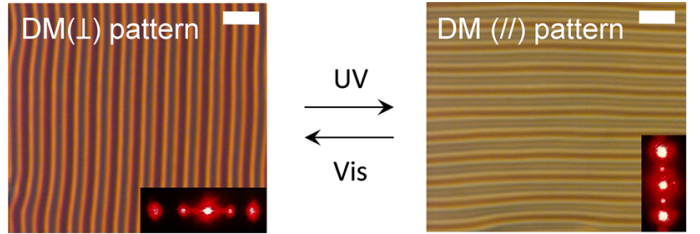

c)

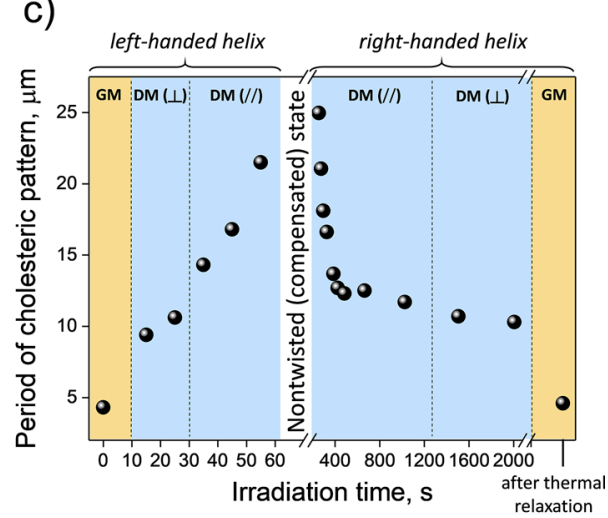

d)

c)

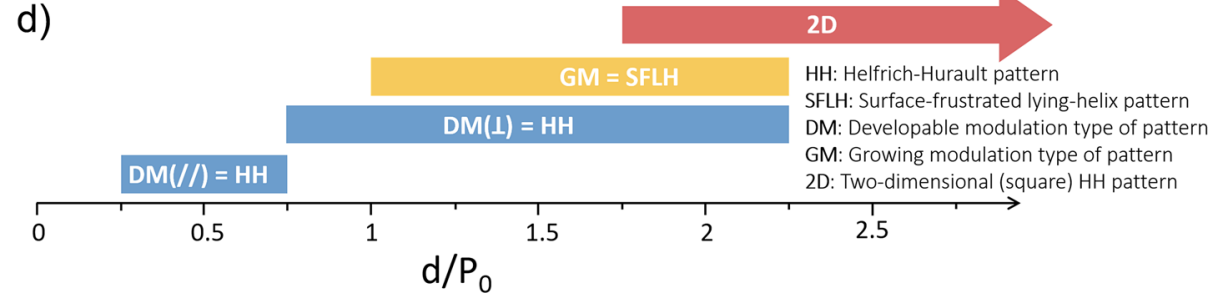

Figure 3. (a) Polarized optical images of $\mathrm{DM}(\|), \mathrm{DM}(\perp)$, and GM cholesteric electro-induced patterns obtained in a $5 \mu \mathrm{m}$ thin film of mixture 1 . The insets show the corresponding diffraction pictures for a normally incident probe laser beam. Scale bar is $25 \mu \mathrm{m}$. (b) Evolution of cholesteric patterns and their period observed in $5 \mu \mathrm{m}$ cell filled with mixture 1 upon irradiation with UV light $(\lambda=365 \mathrm{~nm})$ and further with visible light $(\lambda=$ $436 \mathrm{~nm})(\mathrm{c})$. The type of patterns $\mathrm{GM}, \mathrm{DM}(\perp), \mathrm{DM}(\|)$ and handedness of cholesteric helix are specified on graphs. (d) Diagram obtained from our experiments and simulations shows the intervals of existence of the electro-induced patterns and indicates the correspondence of pattern's names which can be found in the literature.
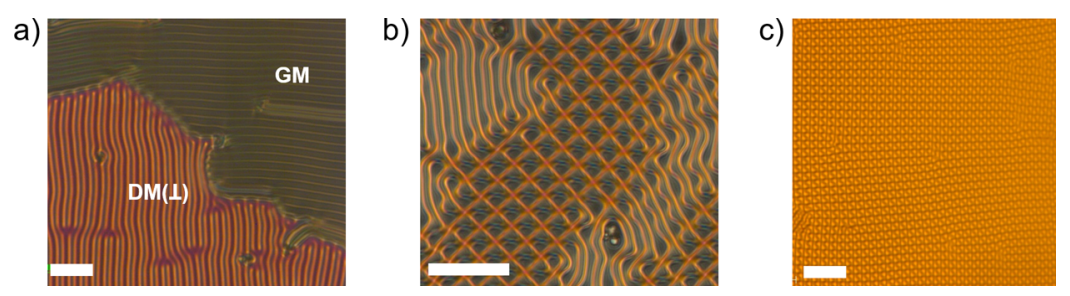

Figure 4. (a) Coexistence of GM and $\mathrm{DM}(\perp)$ cholesteric patterns and (b) growth of $2 \mathrm{D}$ cholesteric pattern in $5 \mu \mathrm{m}$ cell filled with mixture 1; rubbing direction of the cells is horizontal. (c) $2 \mathrm{D}$ cholesteric pattern obtained in a $10 \mu$ m homemade cell $\left(d / P_{0} \approx 7 ; U \approx 3.5 \mathrm{~V}\right)$. Scale bars are 50 $\mu \mathrm{m}$.

described above in the same cholesteric layer by light illumination.

\subsection{Controlling the Geometry of the Periodic} Patterns with Light and Electric Fields. Mixture 1 and mixture 2 were introduced in sandwich glass cells. Next, these thin films were irradiated stepwise with UV light $(\lambda=365 \mathrm{~nm})$ or with visible $(\lambda=436 \mathrm{~nm})$ light. After each exposure to light, an electric field of increasing intensity was applied and periodic in-plane patterns appeared both in mixture 1 (Figure $3 \mathrm{~b}, \mathrm{c}$ ) and in mixture 2 (Figures S3 and S4). In all cases, except for mixture 2 in $5 \mu \mathrm{m}$-gap cells, the ratio $d /\left|P_{0}\right|$ was greater than 1.5 and the structure that appeared first was the GM pattern. For example, in the $5 \mu \mathrm{m}$ cell filled with mixture 1, the GM pattern starts growing at a voltage of about $2.5 \mathrm{~V}$ and has a period of $4.5 \mu \mathrm{m}$.
Upon irradiation of a thin film of mixture 1 with light, the native right-handed cholesteric helix reached the compensated, unwound state and further wound into a left-handed helical structure (Figure 1b). This process was accompanied by a succession of different electro-induced patterns: GM, DM $(\perp$, $M=2), \operatorname{DM}(\|, M=1), 1 \mathrm{D}$ Fréedericksz deformation $(M=0)$, $\operatorname{DM}(\|, M=-1), \operatorname{DM}(\perp, M=-2)$, and again GM (Figure $3 b)$. In fact, at $M \leq-1$, we have observed the mirror counterparts of patterns that have been observed at $M \geq 1$. Thus, irradiation allows not only changing the diffractive pattern period but also rotating the pattern direction by $90^{\circ}$ and inverting the handedness of the helical structure. The conditions for appearance of different structures are discussed in Section 2.5.

The light-induced modifications to the diffractive patterns were reversed by using irradiation with visible light $(\lambda=436$ 
a)

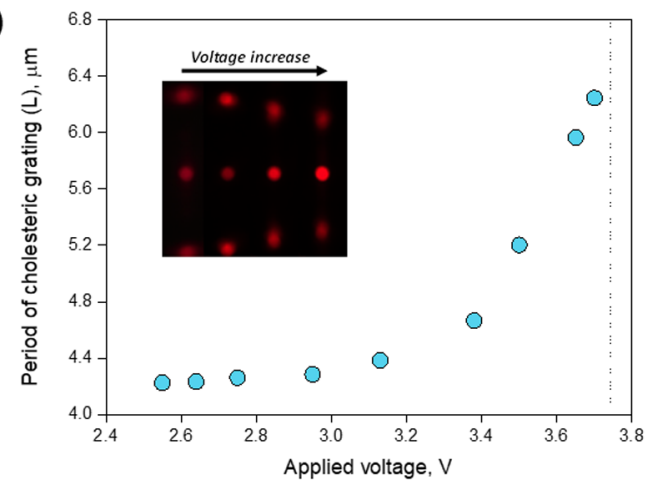

b)

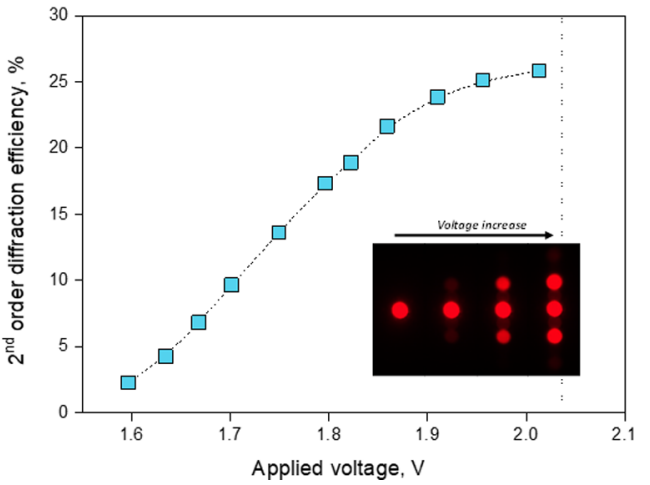

Figure 5. (a) Dependence of the period of a GM pattern and (b) second-order diffraction efficiency of DM( $\|)$ pattern in a $5 \mu \mathrm{m}$ cell with mixture 1 on applied voltage. In both cases, the polarization plane of the probe laser beam is parallel to the pattern stripes. Insets show the evolution of diffraction pictures for the diffraction orders from -2 to 2 with increasing voltage. For all CLC patterns that we observed, the diffracted beams of orders of 1 and -1 , as well as the beams of all other odd diffraction orders, were always very weak compared to the dominant orders. In the insets of figure, they are indistinguishable.

$\mathrm{nm}$ ) that activates the back Z-to-E conversion of MeAzoSorb (Figure 3c). The exact same sequence of structures was observed backward. At the photostationary state promoted by visible light, $P_{0}$ equals to $5 \mu \mathrm{m}$, i.e. the pitch is larger than initially, likely due to the residual presence of Z-MeAzoSorb in the photostationary state. ${ }^{52}$ The complete recovery of the helical pitch and pattern parameters was achieved by thermal Z-to-E relaxation. Notably, irradiation with UV and visible light thus enables tuning the period of $\operatorname{DM}(\|,|M|=1)$ pattern by $100 \%$.

At $d / P_{0} \approx 1(|M|=2)$, it is often possible to observe simultaneously GM and the $\operatorname{DM}(\perp)$ patterns in a state of dynamic equilibrium (Figure 4a). The ratio of the areas occupied by these patterns can be controlled with the voltage. At $d / P_{0} \approx 2$, the $\mathrm{HH}$ deformation is $1 \mathrm{D}$-periodic only at voltages close to $U_{\mathrm{TH}-\mathrm{HH}}$. Increasing the voltage leads to the transformation of the striped pattern into a $2 \mathrm{D}$-periodic one (Figure $4 b$ ). Figure $4 c$ shows a $2 \mathrm{D}$ pattern of a better quality that was obtained at a higher confinement ratio in a homemade electro-optical cell which had no spacer particles in the switched area.

Based on our experimental data and simulation results (see Section 2.5 below) and using the confinement ratio $d / P_{0}$ as a control parameter, we have compiled a diagram of the existence of various periodic patterns within the interval 0.25 $\leq d / P_{0} \leq 2.25$ (Figure 3d), which is of particular interest for practical applications due to a high quality of alignment of the pattern lines. At $d / P_{0}>2.5$ the $\mathrm{HH}$ domain structure has the form of square $2 \mathrm{D}$ pattern, although at $1.75<d / P_{0}<2.5$ both $1 \mathrm{D}$ - and 2D-periodic patterns can exist. In Figure $3 \mathrm{~d}$, we also give the correspondence of the terminology that can be found in the literature. The patterns of the DM type (with both parallel and perpendicular orientation of stripes) correspond to $\mathrm{HH}$ deformations whereas the GM type corresponds to the SFLH pattern.

As a cholesteric helix pitch can be controlled by electric field (but in drastically narrower range than one enabled by light), some of the parameters of the obtained patterns can be tuned as well. The applying voltage to CLC layer causes the helix pitch untwisting. This phenomenon was successfully used in beam steering devices for the tuning of period of GM gratings $L$ which increases with increasing $U .^{19,47}$ We have also addressed this possibility to tune the parameters of patterns in our experimental cells. Figure 5a shows a typical $L$ versus $U$ curve for GM patterns. The increase of $L$ with $U$ is clearly seen from the change of the diffraction pictures (inset in Figure 5a). The width of the voltage interval of GM pattern existence $\left(U_{\mathrm{UT}}-U_{\mathrm{TH}-\mathrm{GM}}\right)$ is about $1.2 \mathrm{~V}$ with the growth of grating period by $50 \%$. The critical field $U_{\mathrm{UT}}$ at which the pattern disappeared is marked in Figure 5a by the vertical dashed line and equals to $3.7 \mathrm{~V}$. $\mathrm{DM}(\|)$ patterns exist in a narrower voltage interval $(\sim 0.4 \mathrm{~V})$ than $\mathrm{GM}$ gratings. Increasing the applied field in the case of $\mathrm{DM}(\|)$ patterns results mainly in an increase of the diffraction efficiency (Figure $5 b$ ) while changes in $L$ are insignificant. $\mathrm{DM}(\perp)$ patterns exhibited a similar behavior.

The study of optical properties of cholesteric diffractive patterns revealed that GM and $\operatorname{DM}(\|)$ ones are commonly more effective for light linearly polarized parallel to the rubbing direction than for light with the orthogonal polarization. To a greater extent, this effect is exhibited by GM patterns. The case of $\operatorname{DM}(\perp)$ is characterized by a less pronounced dependence of diffraction efficiency on the direction of polarization plane of the probe beam; however, the pattern demonstrates a high degree of selectivity to circularly polarized light. Experimental data and computer simulations of optical properties of CLC diffractive patterns can be found in Supporting Information (Figure S6).

Thus, the ability to easily tune $P_{0}$ by light and voltage applied together with reversible inversion of the CLC mixture chirality, significantly widens the space of achievable characteristics of the cholesteric diffractive patterns. The developed CLC mixtures allow obtaining, in a standard electro-optical cell of a fixed thickness, all types of $1 \mathrm{D}$ and $2 \mathrm{D}$ cholesteric in-plane patterns and controlling their parameters efficiently.

2.4. Structuring of the Cholesteric Patterns. One of the current challenges for optical materials is the spatial structuring of diffractive patterns. There are few ways to fabricate structured cholesteric patterns by localized irradiation which are based on using photo-alignment coatings, ${ }^{24}$ hybrid-aligned cholesteric layers, ${ }^{38}$ or stepwise stabilization of each diffractive pattern by polymer networks. ${ }^{21,30}$ Here we propose an alternative strategy where we exploit the high thermal stability of the Z-MeAzoSorb in order to tune the helical pitch locally, with even the possibility to invert handedness.

Therefore, a $5 \mu \mathrm{m}$ thin film of cholesteric mixture 1 was irradiated with a focused UV light beam (the light spot diameter is about $0.5 \mathrm{~mm}$ ) having a near Gaussian intensity 

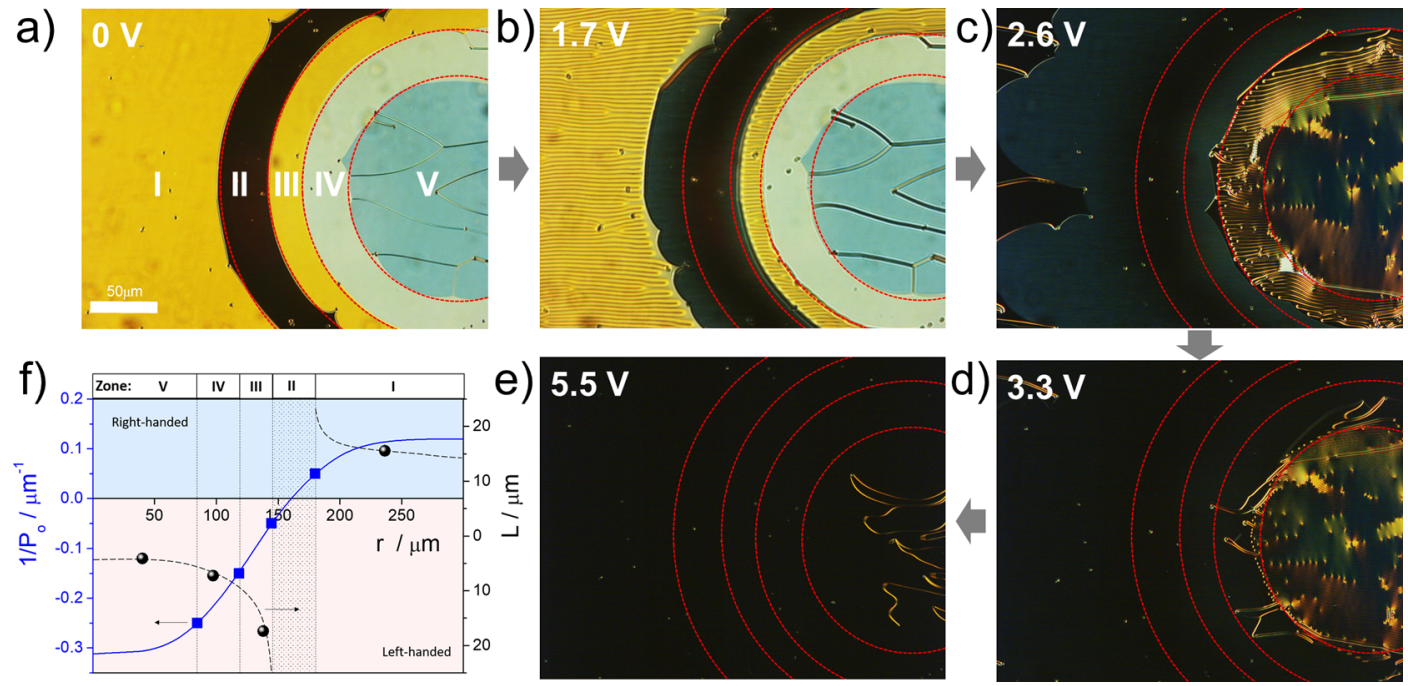

Figure 6. images of a $5 \mu \mathrm{m}$ layer of mixture 1 locally irradiated with UV light (beam diameter $0.5 \mathrm{~mm}$; light dose $\sim 150 \mu \mathrm{J}$ ) under applied voltage (a) $U=0$, (b) 1.7, (c) 2.6, (d) 3.3, and (e) $5.5 \mathrm{~V}$. The boundaries of the Grandjean zones in the field-off state are shown by dashed lines. (f) Tentative dependence of $P_{0}$ and grating period $L$ of electro-induced patterns on the distance, $r$, from the center of zone $\mathrm{V}$.

distribution (Figure 6a-e). The irradiation produces a radial spatial distribution of $P_{0}$ and leads to the appearance of three annular zones with $M$ equal to 0 (zone II; $\Phi=0$ ), -1 , (zone III, $\Phi=-180^{\circ}$ ), and -2 (zone IV, $\Phi=-360^{\circ}$ ) as well as the appearance of a circular zone with $M=-3$ (zone V, $\Phi=$ $\left.-540^{\circ}\right)$, separated from each other and from the background zone I with $M=1\left(\Phi=180^{\circ}\right)$ by Grandjean-Cano disclinations. ${ }^{53}$ Zones III, IV, and V have a left-handed twisted structure, as opposed to zone I. The dependence of $P_{0}$ on the distance from the center of zone $\mathrm{V}$ is shown in Figure $6 \mathrm{f}$. Zone II with $M=0$, which corresponds to nontwisted or compensated state, appears black because the transmission axis of the analyzer in Figure 6 is parallel to the rubbing direction and perpendicular to that of the polarizer. Applying a voltage of $1.7 \mathrm{~V}$ results in the simultaneous formation of enantiomorphous $\mathrm{DM}(\|)$ patterns in zones I and III (Figure $6 \mathrm{~b})$. Further increase of the voltage induces the GM patterns in zones IV and V (Figure 6c) and erasure of the $\mathrm{DM}(\|)$ pattern. At $U=3.5 \mathrm{~V}$, only the GM grating is still visible (Figure 6d). When the voltage reaches $U=5 \mathrm{~V}$, all the gratings have disappeared (Figure 6e; for all zones $U_{\mathrm{UT}}<5 \mathrm{~V}$ ) and the thin film displays a quasi-homeotropic organization through the entire illuminated area. Switching off the voltage returns the system back to the initial state (Figure 6a). The typical values of the pattern period for each zone are shown by black circles in Figure 6f. The $\mathrm{DM}(\perp)$ patterns were never observed, likely because small irregularities of the layer allow the GM domains to appear at voltages below the threshold for $\mathrm{HH}$-deformation $\left(U_{\mathrm{TH}-\mathrm{GM}}<U_{\mathrm{TH}-\mathrm{HH}}\right)$, and at $U=U_{\mathrm{TH}-\mathrm{HH}}$ both the zones feature the GM pattern. We conclude that localized irradiation of the sample allows spatially resolved switching of the type of electro-induced patterns and their parameters, as well as locally inverting the chirality of supramolecular helices.

Dramatic switching between patterns can also be photoinduced in a circularly oriented CLC layers (Figure 7). The thin layer of mixture 1, pre-exposed to ambient light shows radial orientation of electro-induced stripes which correspond to $\mathrm{DM}(\perp)$ mode $(\mathrm{HH}$-stripes oriented perpendicular to the rubbing direction). After exposure to UV light, the sample forms a circular pattern upon applying voltage, the stripes of which go along the local rubbing direction $[\mathrm{DM}(\|)$ mode].
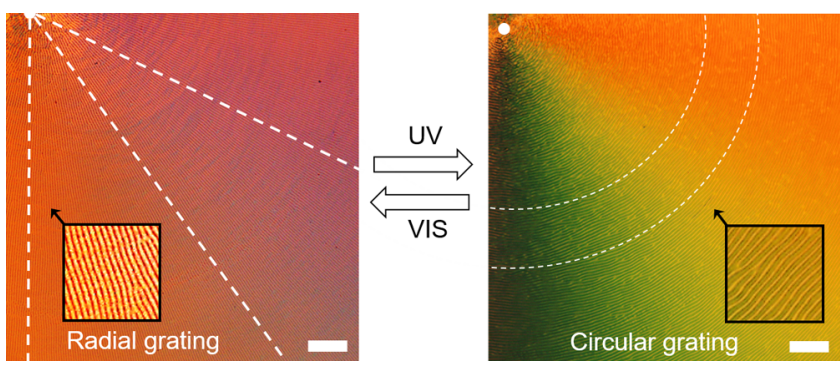

Figure 7. Photoswitching between radial and circular patterns. The 6 $\mu \mathrm{m}$-gap cell with circularly rubbed polyimide orientation layer filled with mixture 1 . Scale bars are $100 \mu \mathrm{m}$. Insets show zoomed areas (100 $\left.\times 100 \mu \mathrm{m}^{2}\right)$ of the textures. Dashed lines display alignment of electroinduced fringes. White dot corresponds to the center of sample.

Exposure to visible light switches the structure back to the radial one.

2.5. Numerical Simulations for the Electro-Induced In-Plane Patterns. We have used computer simulations to further elucidate the parameters that allow for a specific pattern to be formed. An approach proposed by Tsoy et al. ${ }^{54,55}$ was used to calculate the threshold voltage of 1D-periodic $\mathrm{HH}$ deformation, $U_{\mathrm{TH}-\mathrm{HH}}$, the equilibrium period, $L$, and direction of stripes as functions of CLC layer parameters (Figure 8a,b). The equilibrium values of $L$ are shown for applied voltage values that equal to the threshold voltage $\left(U=U_{\mathrm{TH}-\mathrm{HH}}\right)$ and slightly above it $\left(U_{\mathrm{TH}-\mathrm{HH}}+0.01 \mathrm{~V}\right.$ and $\left.U_{\mathrm{TH}-\mathrm{HH}}+0.05 \mathrm{~V}\right)$.

We observe that the range of threshold voltages is shifted toward higher values with increasing $|M|$, which is a characteristic feature of $\mathrm{HH}$-deformation. ${ }^{40,42}$ Experimental values of $U_{\text {TH-GM }}$ (Figure S5) fall within the interval of threshold voltages of $\mathrm{HH}$-patterns for CLC layers with stable field-off configurations with $|M|=2\left(U_{\mathrm{TH}-\mathrm{HH}} \approx 2.5 \mathrm{~B}\right)$ and $|M|$ $=3\left(U_{\mathrm{TH}-\mathrm{HH}} \approx 3.5 \mathrm{~B}\right)$. Therefore, when configurations with $|M|$ $=2$ and $|M|=1$ were stable in the field-off state, we have managed to obtain stable DM pattern. Experimental values of voltage between indium tin oxide layers $\left(U_{\mathrm{TH}-\mathrm{A}}\right)$ at which periodical deformation becomes visible are shown by symbols in Figure 8a. Values of $U_{\text {TH-A }}^{56}$ for all tested cells are in the ranges $2.6-2.85 \mathrm{~V}$ at $|M|=2$, and $1.65-1.9 \mathrm{~V}$ at $|M|=1$, which 
a)

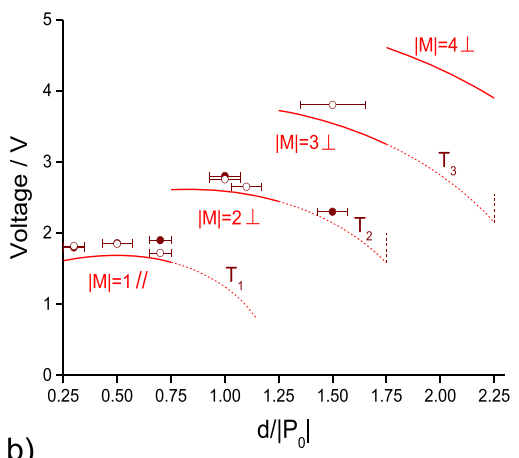

b)

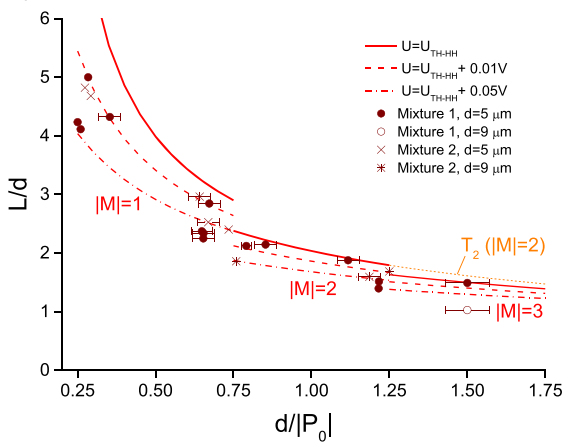

c)

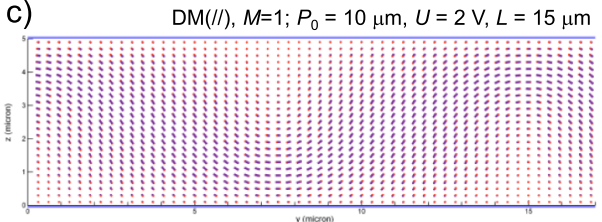

d)

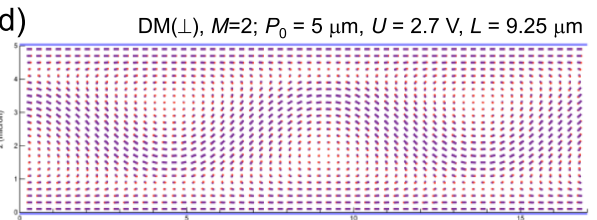

e)

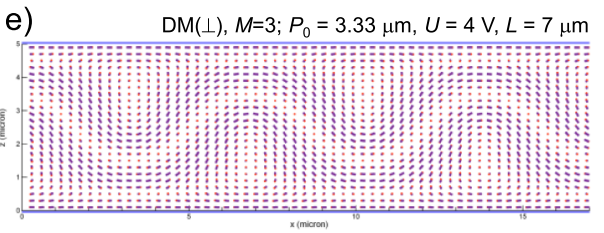

f)

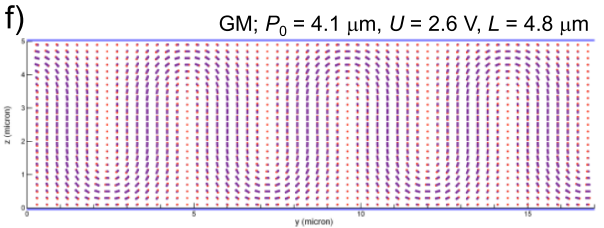

Figure 8. (a) Threshold voltages of HH patterns and types of modulated patterns observed in experimental cells. Red lines show the theoretical values of the threshold voltage $U_{\mathrm{TH}-\mathrm{HH}}$. The solid parts of the red lines correspond to the situation when the planar pattern with the given $|M|$ is equilibrium at zero voltage. The dashed part of the red line is for the situation when the planar pattern with the given $|M|$ is transient (metastable) at zero voltage and has an overstretched cholesteric helix (zones $\mathrm{T}_{1}, \mathrm{~T}_{2}$, and $\mathrm{T}_{3}$ ). Symbols $\|$ and $\perp$ show the theoretical prediction for the direction of stripes with respect to the rubbing direction. Solid and open circles indicate experimental values of the threshold voltage $U_{\mathrm{TH}-\mathrm{A}}$ for 5 and $9 \mu \mathrm{m}$ gap cells, respectively. (b) Near-threshold period of striped $\mathrm{HH}$ patterns. Red lines show the theoretical values of $L / d$ for the equilibrium state in the situation when the planar pattern with the given $M$ is equilibrium at zero voltage. The orange dash line corresponds to zone $\mathrm{T}_{2}$ in Figure $8 \mathrm{a}$. Experimental values of $L / d$ are shown by symbols. Arrow indicates that $2 \mathrm{D}$ patterns can also be obtained at higher $d / P_{0}$ ratios. (c-e) LC orientation in transverse cross-sections of stripes of HH patterns and a SFLH patterns (f). The rubbing direction is along the $x$ axis. Thickness $d$ of the LC layer is $5 \mu \mathrm{m}$.

is in good agreement with theoretical predictions for $U_{\mathrm{TH}-\mathrm{HH}}$. The threshold voltages of $\mathrm{HH}$-deformation at $d /\left|P_{0}\right|>1.25$ for our samples were significantly higher than the threshold voltages for appearance of GM patterns. Nevertheless, we have observed fairly stable patterns of $\mathrm{HH}$-deformation at $1.25<d / 1$ $P_{0} \mid<2.25$, but for transient planar states (see below) rather than for the ground states in zone $\mathrm{T}_{2}$ (Figure 8a), where $U_{\mathrm{TH}-\mathrm{HH}}<U_{\mathrm{TH}-\mathrm{GM}}$, and in zone $\mathrm{T}_{3}$, where $\mathrm{U}_{\mathrm{TH}-\mathrm{HH}}$ is close to $\mathrm{U}_{\mathrm{TH}-\mathrm{GM}}$. In the $\mathrm{T}_{2}$ mode the deformation was striped, i.e. it was periodic in one dimension only. In the $\mathrm{T}_{3}$ mode, the $\mathrm{HH}$ deformation was $1 \mathrm{D}$-periodic only at voltages close to $U_{\mathrm{TH}-\mathrm{HH}}$. The changes of the near-threshold period of $\mathrm{HH}$-pattern with variation of $P_{0}$ were the same for mixtures 1 and 2 and are well in line with the theoretical predictions (Figure $8 \mathrm{~b}$ ).

The threshold voltage for the appearance of GM patterns, $U_{\mathrm{TH}-\mathrm{GM}}$, depends not only on the LC parameters but also on the concentration and character of inclusions (such as spacer particles) and other factors of non-uniformity of the LC layer (see, e.g., ref 23). When $U_{\mathrm{TH}-\mathrm{GM}}<U_{\mathrm{TH}-\mathrm{HH}}$, a $\mathrm{HH}$ pattern does not appear at all or may be observed while the GM pattern is growing. When $U_{\mathrm{TH}-\mathrm{GM}}>U_{\mathrm{TH}-\mathrm{HH}}$, the GM pattern displaces the $\mathrm{HH}$ structure with increasing voltage. For the studied CLC cells, the threshold voltage $\mathrm{U}_{\mathrm{TH}-\mathrm{GM}}$ ranged from 2.5 to $3 \mathrm{~V}$ and had the tendency to slightly decrease with increasing $\left|P_{0}\right|$.

The period $L$ of GM patterns is close to $P_{0}$ (typically, $L / P_{0}$ $\approx 1.2$, see, e.g. ref 13 ) at voltage values close to $U_{\mathrm{TH}-\mathrm{GM}}$ and increases with increasing voltage. The latter is often interpreted as unwinding of the cholesteric helix. Experimental points $(L$, $U_{\text {TH-GM }}$ ) obtained at different stages of phototreatment of tested cells are plotted in Figure S5. The range of the fieldinduced variations of $L$ for GM patterns is generally significantly wider than for $\mathrm{HH}$ patterns.

Figure $8 \mathrm{c}-\mathrm{e}$ shows the typical configuration of the LC director field in transverse cross-sections of stripes for layers with $M=1,2$, and 3 . The director fields were computed using the MOUSE-LCD2D modeling system. ${ }^{57}$

To the best of our knowledge, in layers with $|M|=1$, the DM pattern is the only possible in-plane periodic deformation. At I $M l \geq 2$, a GM pattern (a SFLH structure, Figure 8f) may appear, as it is energetically more favorable. In the middle of a GM layer, the LC configuration is helicoidal with the helix axis parallel to the layer surfaces (Figure $8 \mathrm{f}$ ) and commonly perpendicular to the rubbing direction. In the near-surface regions, where the LC adapts to the boundary conditions, the LC configuration is more complicated. Figure $8 \mathrm{f}$ shows the socalled "snake-type" configuration, ${ }^{58}$ one of the possible SFLH patterns, which looks like an overdistorted $\mathrm{HH}$ configuration for $M=1$ (cf. Figure $8 \mathrm{c}$ ). It should be stressed that the equilibrium field-off (ground-state) structure corresponding to that shown in Figure $8 \mathrm{f}$ has $M=2$, so that the ground-state structure and the SFLH pattern are topologically distinct. The transformation of an ideal defect-free planar ground-state structure to an SFLH pattern with increasing voltage is impossible because of a high energetic barrier between these 
states. Irregularities of real systems make this transformation possible and often very easy, nucleating the growth of line defects in direction parallel to the rubbing.

In thin cholesteric films with $|M| \geq 2$, one can observe $\mathrm{HH}$ deformation not only as a distortion of the ground planar structure, but also the LC layer can be carried to a metastable (transient) planar state with a lesser $|M|$ by a certain voltage switching sequence. ${ }^{59}$ In this state, the actual pitch $P$ is too large to satisfy eq 4 , i.e. the cholesteric helix is overstretched. By increasing the voltage one can obtain a stable in-plane periodic pattern, which can be regarded as a result of the $\mathrm{HH}$ deformation of the transient structure. A remarkable example of using the $\mathrm{HH}$ deformation of the transient planar structure for switching the direction of stripes in CLC pattern was given by Jau et al. ${ }^{59}$ In that example, the ground state had $|M|=2$, and the $\mathrm{HH}$ deformation of the transient configuration $(|M|=$ 1) gave a pattern similar to that shown in Figure $8 \mathrm{f}$, which is in fact a SFLH pattern. The dashed curves for $|M|=1,2$, and 3 in Figure 8a correspond to sections for which the planar pattern is observed only transiently. In all cases, we observe that the threshold voltage of $\mathrm{HH}$ deformation for the transient state is significantly lower than for the ground state. The example of $\mathrm{HH}$-deformation of a transient planar structure from ref 59 corresponds to zone $T_{1}$ in Figure 8 a.

Another salient feature of this system is the $\mathrm{HH}$ pattern at I $M \mid=3$ (Figure 8e). In many papers, one can find the proposition that the direction of $\mathrm{HH}$ stripes is always perpendicular to the direction of LC molecules in the midplane of the LC layer in the field-off state. This rule is not universally true. In our case, as well as in examples provided by Chigrinov et al., ${ }^{42}$ it is violated at $|M|=3$ (see Figure $8 \mathrm{e}$; in the undistorted state, the LC molecules in the middle layer and the stripe direction are perpendicular to the rubbing direction and parallel to each other), which is in complete agreement with experiment.

Overall, the proposed methods of comprehensive computer simulation of electro-induced patterns allow extracting parameters such as threshold voltage, period, LC director field and optical properties, and go well in line with experimental observations providing a reliable predictive tool for patterns based on chiral LCs.

\section{CONCLUSIONS}

The light-controlled operation of chiral switches is successfully converted into tuning periodic in-plane patterns in thin films of CLCs, that can be used as tunable diffraction gratings. Adjustability of the helical structures enables fine control over their optical properties. Specifically, irradiating these lightresponsive cholesteric helices with light allows switching between two fundamentally different periodic patterns: $\mathrm{HH}$ deformation (or DM pattern) represented by periodic bending of cholesteric planes, and SFLH structure (or GM pattern). Depending on the conditions of irradiation, the period of the patterns can be gradually varied from $4.5 \mu \mathrm{m}$ to almost $30 \mu \mathrm{m}$, for both left- and right-handed helical structures. The period of patterns and their diffraction efficiency can be additionally controlled by the voltage applied. The direction of pattern lines can be photoswitched in-plane between two mutually orthogonal directions enabling switching between patterns with radial and circular stripes alignment. Moreover, we demonstrate the unprecedented possibility to switch between linear and square patterns. The good thermal stability of the Zform of the chiral switch also allows performing local structuring. A set of reliable methods for computer simulation of electro-induced CLC gratings is presented, as computer simulations can simplify the search for optimal materials, design parameters and operating modes of devices, as well as provide an assessment of achievable output characteristics. Overall, the electropatterning of light-responsive chiral nematic LCs allows creating versatile in-plane patterns, which can be used as efficient diffraction gratings. These dynamic layers show clear potential for application to adaptive optics, and we also envision their use as active media for colloid- and biosciences.

\section{EXPERIMENTAL SECTION}

4.1. Materials. Low-molar-mass LC ZLI1132 produced by Merck $\left(T_{\text {iso }} \approx 71{ }^{\circ} \mathrm{C}\right)$ was exploited as a host nematic matrix. Chiral dopant LM36 and photoswitch MeAzoSorb were used for the induction of cholesteric mesophase in ZLI1132. MeAzoSorb and LM36 were synthesized as described previously. ${ }^{37,38}$ The cholesteric mixtures were prepared by dissolving all components in chloroform. Then, the solvent was slowly evaporated and residue was dried in vacuum at 50 ${ }^{\circ} \mathrm{C}$ for several hours. Mixture 1 and mixture 2 were prepared according to the following formulation ZLI1132/MeAzoSorb/LM36 (in wt \%): 95.5/1.5/3 (mixture 1), 99.3/0.7/0.0 (mixture 2).

As mixture 1 and mixture 2 differ from one another only by chiral dopants in small concentrations, they are very similar in their optical properties as well as in their behavior in electric fields. In all calculations for these mixtures, we used the following values of elastic and dielectric constants: $K_{11}=1.01 \times 10^{-6} \mathrm{dyn}, K_{22}=5.6 \times 10^{-7} \mathrm{dyn}$, and $K_{33}=1.97 \times 10^{-6} \mathrm{dyn}$; low-frequency $(1 \mathrm{kHz})$ principal permittivities $\varepsilon_{\|}=15$ and $\varepsilon_{\perp}=4.7$ (data for ZLI1132 from Merck).

4.2. Sample Preparation. Commercial 5 and $9 \mu \mathrm{m}$-gap electrooptical cells with planar boundary conditions (Instec, Inc.) were filled by capillary forces, at room temperature. A planar cholesteric texture of high quality was obtained, as verified by polarized optical microscopy.

4.3. Light Source. The samples were irradiated at room temperature with the collimated light of a high-pressure $\mathrm{Hg}$ lamp [HBO lamp, $100 \mathrm{~W}$, "Osram" equipped with interference filters with peak wavelengths of $365 \mathrm{~nm}\left(I \approx 0.4 \mathrm{~mW} / \mathrm{cm}^{2}\right)$ and $436 \mathrm{~nm}(I \approx 0.8$ $\left.\left.\mathrm{mW} / \mathrm{cm}^{2}\right)\right]$. The intensity of light was measured by a LaserMate-Q (Coherent) intensity meter.

4.4. Optical Measurements. The Grandjean-Cano method was used in order to estimate the $\beta$ values of chiral dopants and the pitch of CLCs (wedge cells with $\tan \theta=0.0115$ were purchased from E.H.T. Co. Ltd.). A laser diode $(\lambda=660 \mathrm{~nm}, 3.5 \mathrm{~mW})$ was used to monitor the optical properties of the gratings. The probe beam was linearly polarized. Its polarization direction was controlled with achromatic half-wave plate. The laser wavelength was chosen as 660 $\mathrm{nm}$ to prevent photo-induced Z-to-E isomerization of MeAzoSorb dopant (absorbance spectrum is presented in Figure S1). Grating period was measured by means of polarized optical microscopy (AxioPlan 2, "Carl Zeiss").

\section{ASSOCIATED CONTENT}

\section{Supporting Information}

The Supporting Information is available free of charge on the ACS Publications website at DOI: 10.1021/acsami.8b22465.

Absorbance spectra of MeAzoSorb; polarized light microscopy images demonstrating the growth of GM and DM patterns; evolution of cholesteric patterns period of 5 and $9 \mu$ m-gap cells filled with mixture 2 upon light exposure; threshold voltage of GM pattern depending on its period; optical properties of the diffractive patterns; and typical experimental dependences of diffraction efficiencies of cholesteric patterns on 
the polarization state of the incident light and results of modelization (PDF).

\section{AUTHOR INFORMATION}

\section{Corresponding Author}

*E-mail: a.ryabchun@utwente.nl.

\section{ORCID}

Alexander Ryabchun: 0000-0001-9605-3067

Nathalie Katsonis: 0000-0003-1054-6544

\section{Notes}

The authors declare no competing financial interest.

\section{ACKNOWLEDGMENTS}

This work was partially supported by the Russian Foundation for Basic Research (grant no. 19-03-00337) - synthesis of chiral dopants (A.B.), and by Russian Ministry of Education and Science (3.1586.2017/4.6)-modeling (D.Y.). The research is part of the Advanced Research Center for Chemical Building Blocks, ARC CBBC, which is co-founded and cofinanced by the Netherlands Organization for Scientific Research (NWO) and the Netherlands Ministry of Economic Affairs.

\section{REFERENCES}

(1) Browne, W. R.; Feringa, B. L. Making Molecular Machines Work. Nat. Nanotechnol. 2006, 1, 25-35.

(2) Coskun, A.; Banaszak, M.; Astumian, R. D.; Stoddart, J. F.; Grzybowski, B. A. Great Expectations: Can Artificial Molecular Machines Deliver on Their Promise? Chem. Soc. Rev. 2012, 41, 1930.

(3) Chen, J.; Leung, F. K. C.; Stuart, M. C.; Kajitani, T.; Fukushima, T.; van der Giessen, E.; Feringa, B. L. Artificial Muscle-like Function from Hierarchical Supramolecular Assembly of Photoresponsive Molecular Motors. Nat. Chem. 2018, 10, 132-138.

(4) Foy, J. T.; Li, Q.; Goujon, A.; Colard-Itté, J. R.; Fuks, G.; Moulin, E.; Schiffmann, O.; Dattler, D.; Funeriu, D. P.; Giuseppone, N. Dual-light Control of Nanomachines That Integrate Motor and Modulator Subunits. Nat. Nanotechnol. 2017, 12, 540-545.

(5) Orlova, T.; Lancia, F.; Loussert, C.; Iamsaard, S.; Katsonis, N.; Brasselet, E. Revolving Supramolecular Chiral Structures Powered by Light in Nanomotor-doped Liquid Crystals. Nat. Nanotechnol. 2018, 13, 304-308.

(6) Iamsaard, S.; Aßhoff, S. J.; Matt, B.; Kudernac, T.; Cornelissen, J. J. L. M.; Fletcher, S. P.; Katsonis, N. Conversion of Light into Macroscopic Helical Motion. Nat. Chem. 2014, 6, 229-235.

(7) Eelkema, R.; Pollard, M. M.; Vicario, J.; Katsonis, N.; Ramon, B. S.; Bastiaansen, C. W. M.; Broer, D. J.; Feringa, B. L. Nanomotor rotates microscale objects. Nature 2006, 440, 163.

(8) White, T. J.; Broer, D. J. Programmable and Adaptive Mechanics with Liquid Crystal Polymer Networks and Elastomers. Nat. Mater. 2015, 14, 1087-1098.

(9) Kumar, K.; Knie, C.; Bléger, D.; Peletier, M. A.; Friedrich, H.; Hecht, S.; Broer, D. J.; Debije, M. D.; Schenning, A. P. A Chaotic Selfoscillating Sunlight-driven Polymer Actuator. Nat. Commun. 2016, 7, 11975.

(10) Morrow, S. M.; Bissette, A. J.; Fletcher, S. P. Transmission of Chirality Through Space and Across Length Scales. Nat. Nanotechnol. 2017, 12, 410-419.

(11) Chilaya, G. Cholesteric Liquid Crystals: Properties and Applications; LAP Lambert Academic Publishing: Saarbrücken, Germany, 2013; pp 3-112.

(12) Ryabchun, A.; Bobrovsky, A. Cholesteric Liquid Crystal Materials for Tunable Diffractive Optics. Adv. Opt. Mater. 2018, 6, 1800335.
(13) Subacius, D.; Bos, P. J.; Lavrentovich, O. D. Switchable Diffractive Cholesteric Gratings. Appl. Phys. Lett. 1997, 71, 13501352.

(14) Subacius, D.; Shiyanovskii, S. V.; Bos, P.; Lavrentovich, O. D. Cholesteric Gratings with Field-Controlled Period. Appl. Phys. Lett. 1997, 71, 3323-3325.

(15) Shiyanovskii, S.; Subacius, D.; Voloschenko, D.; Bos, P.; Lavrentovich, O. Cholesteric diffraction devices with a field-controlled grating vector. Proceedings SPIE, 1998; Vol. 3475, pp 56-64.

(16) Wu, J.-J.; Wu, Y.-S.; Chen, F.-C.; Chen, S.-H. Formation of Phase Grating in Planar Aligned Cholesteric Liquid Crystal Film. Jpn. J. Appl. Phys. 2002, 41, L1318-L1320.

(17) Fuh, A. Y.-G.; Lin, C.-H.; Huang, C.-Y. Dynamic Pattern Formation and Beam-steering Characteristics of Cholesteric Gratings. Jpn. J. Appl. Phys. 2002, 41, 211-218.

(18) Zheng, Z.-g.; Li, Y.; Bisoyi, H. K.; Wang, L.; Bunning, T. J.; Li, Q. Three-dimensional Control of the Helical Axis of a Chiral Nematic Liquid Crystal by Light. Nature 2016, 531, 352-356.

(19) Jau, H.-C.; Li, Y.; Li, C.-C.; Chen, C.-W.; Wang, C.-T.; Bisoyi, H. K.; Lin, T.-H.; Bunning, T. J.; Li, Q. Light-Driven Wide-Range Nonmechanical Beam Steering and Spectrum Scanning Based on a Self-Organized Liquid Crystal Grating Enabled by a Chiral Molecular Switch. Adv. Opt. Mater. 2015, 3, 166-170.

(20) Bisoyi, H. K.; Li, Q. Light-driven Liquid Crystalline Materials: From Photo-induced Phase Transitions and Property Modulations to Applications. Chem. Rev. 2016, 116, 15089.

(21) Li, W.-S.; Shen, Y.; Chen, Z.-J.; Cui, Q.; Li, S.-S.; Chen, L.-J. Demonstration of Patterned Polymer-stabilized Cholesteric Liquid Crystal Textures for Anti-counterfeiting Two-dimensional Barcodes. Appl. Opt. 2017, 56, 601-606.

(22) Gvozdovskyy, I. Electro- and Photoswitching of Undulation Structures in Planar Cholesteric Layers Aligned by a Polyimide Film Possessing Various Values of the Anchoring Energy. Liq. Cryst. 2018, $45,536-552$.

(23) Senyuk, B.; Smalyukh, I.; Lavrentovich, O. Electricallycontrolled Two-dimensional Gratings Based on Layers Undulations in Cholesteric Liquid Crystals. Proceedings SPIE, 2005, Vol. 5936, pp 59360W1-59360W9.

(24) Ma, L.-L.; Li, S.-S.; Li, W.-S.; Ji, W.; Luo, B.; Zheng, Z.-G.; Cai, Z.-P.; Chigrinov, V.; Lu, Y.-Q.; Hu, W.; Chen, L.-J. Rationally Designed Dynamic Superstructures Enabled by Photoaligning. Adv. Opt. Mater. 2015, 3, 1655.

(25) Zheng, Z.-g.; Zola, R. S.; Bisoyi, H. K.; Wang, L.; Li, Y.; Bunning, T. J.; Li, Q. Controllable Dynamic Zigzag Pattern Formation in a Soft Helical Superstructure. Adv. Mater. 2017, 29, 1701903.

(26) Yeh, H.-C.; Chen, G.-H.; Lee, C.-R.; Mo, T.-S. Photoinduced Two-dimensional Gratings Based on Dye-doped Cholesteric Liquid Crystal Films. J. Chem. Phys. 2007, 127, 141105.

(27) Yao, I.-A.; Wu, J.-J.; Chen, S.-H. Observation of Electric-fielddriven Modulated Textures in Cholesteric Liquid Crystal Cells. Jpn. J. Appl. Phys. 2004, 43, 6278-6279.

(28) Ryabchun, A.; Bobrovsky, A.; Stumpe, J.; Shibaev, V. Electroinduced Diffraction Gratings in Cholesteric Polymer with Phototunable Helix Pitch. Adv. Opt. Mater. 2015, 3, 1462-1469.

(29) Kang, S. W.; Sprunt, S.; Chien, L. C. Structure and Morphology of Polymer-stabilized Cholesteric Diffraction Gratings. Appl. Phys. Lett. 2000, 76, 3516-3518.

(30) Li, W.-S.; Ma, L.-L.; Gong, L.-L.; Li, S.-S.; Yang, C.; Luo, B.; $\mathrm{Hu}, \mathrm{W}$; Chen, L.-J. Interlaced Cholesteric Liquid Crystal Fingerprint Textures via Sequential UV-induced Polymer-stabilization. Opt. Mater. Express 2016, 6, 19-28.

(31) Varney, M. C. M.; Zhang, Q.; Senyuk, B.; Smalyukh, I. I. Selfassembly of Colloidal Particles in Deformation Landscapes of Electrically Driven Layer Undulations in Cholesteric Liquid Crystals. Phys. Rev. E 2016, 94, 042709.

(32) Aßhoff, S. J.; Lancia, F.; Iamsaard, S.; Matt, B.; Kudernac, T.; Fletcher, S. P.; Katsonis, N. High-Power Actuation from Molecular Photoswitches in Enantiomerically Paired Soft Springs. Angew. Chem., Int. Ed. 2017, 56, 3261-3265. 
(33) Liu, D.; Bastiaansen, C. W. M.; den Toonder, J. M. J.; Broer, D. J. Photo-Switchable Surface Topologies in Chiral Nematic Coatings. Angew. Chem., Int. Ed. 2012, 51, 892-896.

(34) Katsonis, N.; Lacaze, E.; Ferrarini, A. Controlling Chirality with Helix Inversion in Cholesteric Liquid Crystals. J. Mater. Chem. 2012, 22, 7088-7097.

(35) Bisoyi, H. K.; Bunning, T. J.; Li, Q. Stimuli-Driven Control of the Helical Axis of Self-Organized Soft Helical Superstructures. Adv. Mater. 2018, 30, 1706512.

(36) Bisoyi, H. K.; Li, Q. Light-directed Dynamic Chirality Inversion in Functional Self-organized Helical Superstructures. Angew. Chem. Int. Ed. 2016, 55, 2994-3010.

(37) Bobrovsky, A.; Ryabchun, A.; Cigl, M.; Hamplová, V.; Kašpar, M.; Hampl, F.; Shibaev, V. New azobenzene-based chiral-photochromic substances with thermally stable Z-isomers and their use for the induction of a cholesteric mesophase with a phototunable helix pitch. J. Mater. Chem. C 2014, 2, 8622-8629.

(38) Ryabchun, A.; Bobrovsky, A.; Stumpe, J.; Shibaev, V. Rotatable Diffraction Gratings Based on Cholesteric Liquid Crystals with Phototunable Helix Pitch. Adv. Opt. Mater. 2015, 3, 1273-1279. . During preparation of the liquid crystal cells, $P_{0}$ increased up to $3.2-4$ $\mu \mathrm{m}$ under the effect of ambient light inducing $\mathrm{E}-\mathrm{Z}$ isomerization with the formation of small amount of Z-isomer. The range of fast and reversible phototuning of $P_{0}$ of mixture 1 is found to be $-0.34 \mu \mathrm{m}^{-1}<$ $1 / P_{0}<0.2 \mu \mathrm{m}^{-1}$

(39) During preparation of the liquid crystal cells, $P_{0}$ increased up to 3.2-4 $\mu \mathrm{m}$ under the effect of ambient light inducing $\mathrm{E}-\mathrm{Z}$ isomerization with the formation of small amount of Z-isomer. The range of fast and reversible phototuning of $P_{0}$ of mixture 1 is found to be $-0.34 \mu \mathrm{m}^{-1}<1 / P_{0}<0.2 \mu \mathrm{m}^{-1}$.

(40) Scheffer, T. J. Electric and Magnetic Field Investigations of the Periodic Gridlike Deformation of a Cholesteric Liquid Crystal. Phys. Rev. Lett. 1972, 28, 593-596.

(41) De Zwart, M.; Van Doorn, C. Z. The Field-induced Square Grid Perturbation in the Planar Texture of Cholesteric Liquid Crystals. J. Phys. Colloq. 1979, 40, C3-278.

(42) Chigrinov, V. G.; Belyaev, V. V.; Belyaev, S. V.; Grebenkin, M. F. Instability of Cholesteric Liquid Crystals in an Electric Field. Sov. Phys. JETP 1979, 50, 994-999.

(43) Helfrich, W. Deformation of Cholesteric Liquid Crystals with Low Threshold Voltage. Appl. Phys. Lett. 1970, 17, 531-532.

(44) Hurault, J. P. Static Distortions of a Cholesteric Planar Structure Induced by Magnetic or ac Electric Fields. J. Chem. Phys. 1973, 59, 2068-2075.

(45) Zhang, L.; Wang, L.; Hiremath, U. S.; Bisoyi, H. K.; Nair, G. G.; Yelamaggad, C. V.; Urbas, A. M.; Bunning, T. J.; Li, Q. Dynamic Orthogonal Switching of a Thermoresponsive Self-Organized Helical Superstructure. Adv. Mater. 2017, 29, 1700676

(46) Hervet, H.; Hurault, J. P.; Rondelez, F. Static One-dimensional Distortions in Cholesteric Liquid Crystals. Phys. Rev. A: At., Mol., Opt. Phys. 1973, 8, 3055-3064.

(47) Lavrentovich, O. D.; Shiyanovskii, S. V.; Voloschenko, D. Fast Beam Steering Cholesteric Diffractive Devices. Proceedings SPIE, 1999; Vol. 3787, pp 149-155.

(48) Gerritsma, C. J.; van Zanten, P. Periodic Perturbations in the Cholesteric Plane Texture. Phys. Lett. A 1971, 37, 47-48.

(49) Rondelez, F.; Arnould, H.; Gerritsma, C. J. Electrohydrodynamic Effects in Cholesteric Liquid Crystals under AC Electric Fields. Phys. Rev. Lett. 1972, 28, 735-737.

(50) Senyuk, B. I.; Smalyukh, I. I.; Lavrentovich, O. D. Undulations of Lamellar Liquid Crystals in Cells with Finite Surface Anchoring Near and Well Above The Threshold. Phys. Rev. E: Stat., Nonlinear, Soft Matter Phys. 2006, 74, 011712.

(51) Kang, S.-W.; Chien, L.-C. Field-induced and Polymer-stabilized Two-dimensional Cholesteric Liquid Crystal Gratings. Appl. Phys. Lett. 2007, 90, 221110.

(52) That is the reason why the stage of DM $(\perp, M=2)$ pattern could only be achieved in cells with $d=5 \mu \mathrm{m}$ by means of visible light irradiation.
(53) Gvozdovskyy, I. Influence of The Anchoring Energy on Jumps of The Period of Stripes in Thin Planar Cholesteric Layers Under The Alternating Electric Field. Liq. Cryst. 2014, 41, 1495-1504.

(54) Tsoy, V. I.; Simonenko, G. V.; Chigrinov, V. G. Dielectric Stripes in Pretilted Supertwisted Layers. Liq. Cryst. 1993, 13, 227231.

(55) This approach is a generalization of the perturbation approach based on the Oseen-Frank elastic theory to the case of arbitrary dielectric anisotropy of LC and nonzero pretilt angles.

(56) The experimental values of $U_{\mathrm{TH}-\mathrm{A}}$ are a bit higher than theoretical values of $U_{\mathrm{TH}-\mathrm{HH}}$ due to voltage drop on polyimide aligning layers (about 5\% for cells with $d=5 \mu \mathrm{m}$ and 3\% for cells with $d=9 \mu \mathrm{m})$ and visual indistinguishability of weak near-threshold deformations.

(57) Yakovlev, D. A.; Tsoy, V. I.; Chigrinov, V. G. 5.4: Advanced Tools for Modeling of 2D-Optics of LCDs. Symp. Dig. Tech. Pap. 2005, 36, 58-61.

(58) Shiyanovskii, S. V.; Smalyukh, I. I.; Lavrentovich, O. D. In Defects in Liquid Crystals: Computer Simulations, Theory and Experiments; Lavrentovich, O. D., Pasini, P., Zannoni, C., Zumer, S., Eds.; Springer: Dordrecht, Netherlands, 2001; Chapter 4, pp 229270.

(59) Jau, H.-C.; Lin, T.-H.; Chen, Y.-Y.; Chen, C.-W.; Liu, J.-H.; Fuh, A. Y.-G. Direction Switching and Beam Steering of Cholesteric Liquid Crystal Gratings. Appl. Phys. Lett. 2012, 100, 131909. 